TITLE:

\title{
Minimal Model of Stochastic Athermal Systems: Origin of Non- Gaussian Noise
}

\section{$\operatorname{AUTHOR(S):~}$}

Kanazawa, Kiyoshi; Sano, Tomohiko G.; Sagawa, Takahiro; Hayakawa, Hisao

\section{CITATION:}

Kanazawa, Kiyoshi ...[et al]. Minimal Model of Stochastic Athermal Systems: Origin of NonGaussian Noise. Physical Review Letters 2015, 114(9): 090601.

\section{ISSUE DATE:}

2015-03-03

URL:

http://hdl.handle.net/2433/198650

RIGHT:

(C) 2015 American Physical Society 


\title{
Minimal Model of Stochastic Athermal Systems: Origin of Non-Gaussian Noise
}

\author{
Kiyoshi Kanazawa, ${ }^{1}$ Tomohiko G. Sano, ${ }^{1}$ Takahiro Sagawa, ${ }^{2}$ and Hisao Hayakawa ${ }^{1}$ \\ ${ }^{1}$ Yukawa Institute for Theoretical Physics, Kyoto University, Kitashirakawa-oiwake cho, Sakyo-ku, Kyoto 606-8502, Japan \\ ${ }^{2}$ Department of Basic Science, The University of Tokyo, Komaba, Meguro-ku 153-8902, Japan \\ (Received 27 July 2014; revised manuscript received 19 August 2014; published 3 March 2015)
}

\begin{abstract}
For a wide class of stochastic athermal systems, we derive Langevin-like equations driven by nonGaussian noise, starting from master equations and developing a new asymptotic expansion. We found an explicit condition whereby the non-Gaussian properties of the athermal noise become dominant for tracer particles associated with both thermal and athermal environments. Furthermore, we derive an inverse formula to infer microscopic properties of the athermal bath from the statistics of the tracer particle. We apply our formulation to a granular motor under viscous friction and analytically obtain the angular velocity distribution function. Our theory demonstrates that the non-Gaussian Langevin equation is the minimal model of athermal systems.
\end{abstract}

DOI: 10.1103/PhysRevLett.114.090601

PACS numbers: 05.70.Ln, 05.10.Gg, 05.40.Fb

Introduction.-Recent developments in experimental techniques have triggered both experimental and theoretical research on fluctuations in various nonequilibrium systems [1-12]. The minimal model for thermally fluctuating systems is the Gaussian Langevin model, which serves as a foundation of recent studies on stochastic thermodynamics [13-26]. Furthermore, athermal fluctuations are also extensively studied in various systems recently [27-41], whose characteristic distinction from thermal fluctuations is a topic of wide interest. In fact, non-Gaussian properties are experimentally observed in various athermal systems [27-41], and thermodynamic properties of such systems have been theoretically studied on the basis of non-Gaussian stochastic models [42-47].

A fundamental question then arises: When and how does non-Gaussianity emerge from microscopic dynamics? While this problem has been well studied in the presence of anomalous fluctuations with asymptotically heavy-tailed distributions of waiting time or jump size [48-50], the origin of non-Gaussianity has not been fully understood with normal fluctuations as is the case for the conventional Langevin systems. Indeed, the conventional coarsegraining theories with normal fluctuations fail to explain non-Gaussian behaviors at leading order, as they always produce the Gaussian noise from the central limit theorem (CLT) [51-54]. To clarify this point, let us review the theory of van Kampen [51,52]. In general nonequilibrium dynamics described by the master equation, the environmental noise is strongly correlated with the state of the system, which implies that the noise is not white (or equivalently, state dependent). In the large system size limit, however, their correlation asymptotically disappears, and the noise distribution becomes Gaussian. This is the origin of the universality of the white Gaussian noise and is true even for the case of genuine nonequilibrium systems without the time-reversal symmetry. Therefore, it is highly nontrivial to explain the origin of the non-Gaussian noise at leading order. We can then rephrase the aforementioned question as follows: When and how can non-Gaussianity emerge against the apparent universality of the CLT?

In this Letter, we answer the above question by developing a new asymptotic expansion of the master equation. To leading order of the system size expansion, we derive a linear non-Gaussian Langevin equation for a wide class of athermal systems under three assumptions: (i) large system size, (ii) strong thermal friction, and (iii) the coexistence of the thermal and athermal noise. Remarkably, nonGaussianity still remains because of the violation of the CLT, although the athermal fluctuation is reduced to the white noise as the system size increases. We also derive an inverse formula to infer the statistics of athermal bath from the probability distribution function (PDF) of the velocity of the system. As a demonstration, we study a granular motor under viscous friction and analytically derive its steady PDF. Furthermore, we obtain a formula to estimate the velocity distribution of the surrounding granular gas from the rotor's PDF that is experimentally observed. This implies that the non-Gaussianity of the PDF plays key roles to infer microscopic properties of the athermal bath.

Setup.-We consider a particle in one-dimensional space attached to thermal and athermal baths (see Fig. 1 as a schematic). For simplicity, we assume that the mass is unity

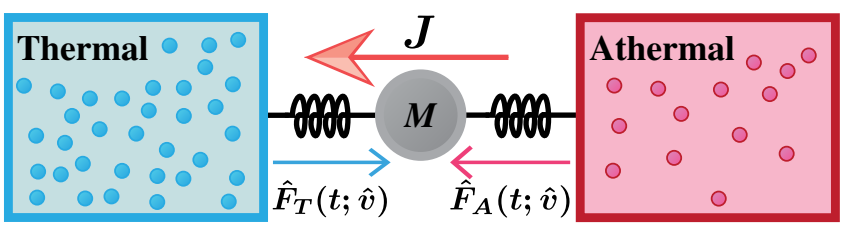

FIG. 1 (color online). Schematic of a system driven by thermal $\hat{F}_{T}(t ; \hat{v})$ and athermal $\hat{F}_{A}(t ; \hat{v})$ forces. There is net energy current $J$ from the athermal to the thermal environment. 
$(M=1)$ and the system obeys Markovian dynamics without mechanical potentials. Then, the dynamical equation for the velocity of the particle $\hat{v}$ is written as

$$
\frac{d \hat{v}}{d t}=\hat{F}_{T}(t ; \hat{v})+\hat{F}_{A}(t ; \hat{v}),
$$

where $\hat{F}_{T}(t ; \hat{v})$ and $\hat{F}_{A}(t ; \hat{v})$ are stochastic forces from the thermal and athermal environments. We denote the ensemble average of stochastic variable $\hat{A}$ by $\langle\hat{A}\rangle$. The thermal force $\hat{F}_{T}(t ; \hat{v})$ is described by the sum of a linear friction and a white Gaussian noise $\hat{F}_{T}(t ; \hat{v})=-\gamma \hat{v}+\sqrt{2 \gamma T} \hat{\xi}_{G}$ with viscous coefficient $\gamma$, temperature $T$, and white Gaussian noise $\hat{\xi}_{G}(t)$ satisfying $\left\langle\hat{\xi}_{G}\left(t_{1}\right)\right\rangle=0$ and $\left\langle\hat{\xi}_{G}\left(t_{1}\right) \hat{\xi}_{G}\left(t_{2}\right)\right\rangle=\delta\left(t_{1}-t_{2}\right)$. Here, we make a critical assumption that $\hat{F}_{A}(t ; \hat{v})$ is a stochastic force characterized by a small positive parameter $\epsilon$ and an $\epsilon$-independent Markovian jump force $\hat{\eta}_{A}(t ; \hat{v})$ such that

$$
\hat{F}_{A}(t ; \hat{v})=\epsilon \hat{\eta}_{A}(t ; \hat{v}),
$$

where $\epsilon$ corresponds to the inverse of the system size in Refs. [51,52] (see the Supplemental Material [55]). The corresponding master equation for the velocity PDF $P(v, t) \equiv P[\hat{v}(t)=v]$ is given by

$$
\begin{aligned}
\frac{\partial P(v, t)}{\partial t}= & \gamma\left[\frac{\partial}{\partial v} v+T \frac{\partial^{2}}{\partial v^{2}}\right] P(v, t) \\
& +\int_{-\infty}^{\infty} d y\left[P(v-y, t) W_{\epsilon}(v-y ; y)\right. \\
& \left.-P(v, t) W_{\epsilon}(v ; y)\right],
\end{aligned}
$$

where $W_{\epsilon}(v ; y)$ is the athermal transition rate from $v$ with velocity jump $y$. The scaling assumption (2) implies that $y$ should be scaled: $\mathcal{Y} \equiv y / \epsilon$. Then, the scaled transition rate $\bar{W}(v ; \mathcal{Y})$ for the scaled velocity jump $\mathcal{Y}$ satisfies

$$
\bar{W}(v ; \mathcal{Y}) d \mathcal{Y}=W_{\epsilon}(v ; y) d y \Leftrightarrow W_{\epsilon}(v ; y)=\frac{1}{\epsilon} \bar{W}\left(v ; \frac{y}{\epsilon}\right),
$$

where $\bar{W}(v ; \mathcal{Y})$ is $\epsilon$ independent, corresponding to the $\epsilon$ independence of $\hat{\eta}_{A}$. Note that the scaling (4) is equivalent to that introduced in Refs. [51,52], and the only difference in the master equation (3) from those in Refs. [51,52] is the presence of the thermal diffusion term $L_{0} \equiv \gamma[(\partial / \partial v) v+$ $T\left(\partial^{2} / \partial v^{2}\right)$ ]. In fact, when the thermal bath is absent $(\gamma=0)$ and $\hat{\eta}_{A}$ is stable [51] around $\hat{v}=0$, the conventional Langevin equation is reproduced [see Fig. 2(a)]:

$$
\frac{d \hat{v}}{d t}=-\gamma_{A} \hat{v}+\sqrt{2 \gamma_{A} T_{A}} \hat{\xi}_{G},
$$

where we have introduced the athermal friction $\gamma_{A} \equiv$ $-\epsilon \alpha_{1}^{\prime}(0)$ and temperature $2 \gamma_{A} T_{A} \equiv \epsilon^{2} \alpha_{2}(0)$ with the Kramers-Moyal coefficient $\alpha_{n}(v) \equiv \int_{-\infty}^{\infty} d \mathcal{Y} \bar{W}(v ; \mathcal{Y}) \mathcal{Y}^{n}$.
We stress that the above theory is applicable to systems without microscopic reversibility, which implies that microscopic irreversibility is not a sufficient condition to derive non-Gaussian models.

Main results.-We next discuss an asymptotic expansion of Eq. (3) in terms of the system size. We make the following three assumptions, which were roughly stated in the introduction: (i) large system size: $\epsilon$ is small; (ii) strong thermal friction: $\gamma \gg \gamma_{A}$ (i.e., $\gamma$ is a positive constant independent of $\epsilon$ ); and (iii) the coexistence of both thermal and athermal noise: the variance in the thermal noise is of the same order as for athermal noise (i.e., $T=\mathcal{T} \epsilon^{2}$ with an $\epsilon$-independent parameter $\mathcal{T}$ ). Condition (i) implies the weak coupling for the athermal bath, which is crucial to truncate the environmental correlation. Condition (ii) implies that the thermal friction is dominant for dissipation, and the athermal force becomes irrelevant to relaxation [see Fig. 2(b)]. We here introduce an appropriate scaled variable to remove the singularity of the small noise expansion: $\mathcal{V} \equiv v / \epsilon$. In the limit $\epsilon \rightarrow 0$, Eq. (3) is reduced to

$$
\begin{aligned}
\frac{\partial \mathcal{P}(\mathcal{V}, t)}{\partial t}= & \gamma\left[\frac{\partial}{\partial \mathcal{V}} \mathcal{V}+\mathcal{T} \frac{\partial^{2}}{\partial \mathcal{V}^{2}}\right] \mathcal{P}(\mathcal{V}, t) \\
& +\int_{-\infty}^{\infty} d \mathcal{Y} \bar{W}(0 ; \mathcal{Y})[\mathcal{P}(\mathcal{V}-\mathcal{Y}, t)-\mathcal{P}(\mathcal{V}, t)]
\end{aligned}
$$

with $\mathcal{P}(\mathcal{V}, t) \equiv \epsilon P(v, t)$. Remarkably, $\bar{W}(0 ; \mathcal{Y})$ is independent of the system's velocity $\hat{\mathcal{V}}$, which implies that the environmental correlation disappears and the athermal fluctuation is reduced to the white noise. Furthermore, the non-Gaussianity still remains after this reduction, as seen from the system's steady distribution [see Fig. 2(b)]. This is the violation of the CLT. Then, Eq. (6) is equivalent to the Langevin-like equation with a white non-Gaussian noise term [52]:

$$
\frac{d \hat{\mathcal{V}}}{d t}=-\gamma \hat{\mathcal{V}}+\sqrt{2 \gamma \mathcal{T}} \hat{\xi}_{G}+\hat{\xi}_{\mathrm{NG}}
$$

where $\hat{\xi}_{\mathrm{NG}}$ is the white non-Gaussian noise with transition rate $\bar{W}(0 ; \mathcal{Y})$. This is the first main result of this Letter. We stress that Eq. (7) is exactly solvable [56]. Indeed, Eq. (6) is reduced to $(d / d s) \tilde{P}_{\mathrm{SS}}(s)=[\Phi(s) / \gamma s] \tilde{P}_{\mathrm{SS}}(s)$ in the steady state, where the convolution in Eq. (6) is simplified by introducing the steady PDF $\mathcal{P}_{\mathrm{SS}}(\mathcal{V}) \equiv \lim _{t \rightarrow \infty} \mathcal{P}(\mathcal{V}, t)$, its Fourier representation $\tilde{P}_{\mathrm{SS}}(s) \equiv \int_{-\infty}^{\infty} d \mathcal{V} e^{i s \mathcal{V}} \mathcal{P}_{\mathrm{SS}}(\mathcal{V})$, and the cumulant function $\Phi(s) \equiv \int_{-\infty}^{\infty} d \mathcal{Y} \bar{W}(0 ; \mathcal{Y})\left(e^{i s \mathcal{Y}}-1\right)-$ $\gamma \mathcal{T} s^{2}$. This equation is easily solved as $\tilde{P}_{\mathrm{SS}}(s)=$ $\exp \left[\int_{0}^{s} d s^{\prime} \Phi\left(s^{\prime}\right) / \gamma s^{\prime}\right]$. The stationary PDF is then given by $\mathcal{P}_{\text {SS }}(\mathcal{V})=\int_{-\infty}^{\infty} d s \exp \left[-i s \mathcal{V}+\int_{0}^{s} d s^{\prime} \Phi\left(s^{\prime}\right) / \gamma s^{\prime}\right] / 2 \pi$. We further obtain the inverse formula of the transition rate $\bar{W}(v ; \mathcal{Y})$ from the stationary PDF as 

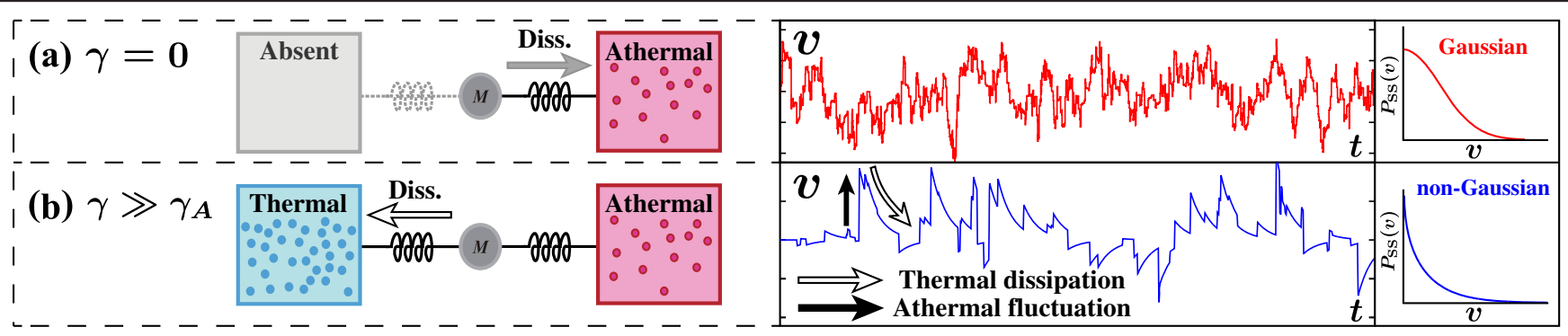

FIG. 2 (color online). Typical trajectories depending on $\gamma$ : (a) For $\gamma=0$, the athermal force is relevant to both fluctuation and dissipation (Diss.), where the non-Gaussianity is irrelevant. (b) For $\gamma \gg \gamma_{A}$, the athermal fluctuation is irrelevant to dissipation because of the dominance of the thermal friction. The athermal non-Gaussianity then becomes relevant.

$$
\bar{W}(0 ; \mathcal{Y})=\gamma \int_{-\infty}^{\infty} \frac{d s}{2 \pi} e^{-i s \mathcal{Y}}\left[\lambda^{*}+\mathcal{T} s^{2}+s \frac{d}{d s} \log \tilde{P}_{\mathrm{SS}}(s)\right]
$$

where we have introduced $\lambda^{*} \equiv \int_{-\infty}^{\infty} d \mathcal{Y} \bar{W}(0 ; \mathcal{Y}) / \gamma$. Note that $\lambda^{*}=-\lim _{s \rightarrow \infty}\left[s(d / d s) \log \tilde{P}_{\mathrm{SS}}(s)+\mathcal{T} s^{2}\right]$ according to the Riemann-Lebesgue lemma [57]. This is the second main result of this Letter, which connects the microscopic transition rate $\bar{W}(v ; \mathcal{Y})$ and the observable $\mathcal{P}_{\mathrm{SS}}(\mathcal{V})$. Equation (8) is derived from the inverse Fourier transformation of the definition of the cumulant function as $\bar{W}(0 ; \mathcal{Y})=\gamma \int_{-\infty}^{\infty} d s e^{-i s \mathcal{Y}}\left[\lambda^{*}+\mathcal{T} s^{2}+\Phi(s) / \gamma\right] / 2 \pi$ and the relation $\Phi(s) / \gamma=s(d / d s) \log \tilde{P}_{\mathrm{SS}}(s)$. The effectiveness of Eq. (8) will be demonstrated later by an example of a granular motor. We note that our formulation is applicable to the small noise expansion for a single multiplicative Lévy noise [58]. We also note that our formulation reduces to the independent kick model [32-35] in the limit $\gamma \rightarrow \infty$ (see the Supplemental Material [55]).

Equation (7) does not satisfy the detailed balance condition because there is net energy current from the athermal to the thermal environment as $J=\langle d \hat{Q} / d t\rangle_{\mathrm{SS}}=$ $K_{2}>0$, where we have introduced the second cumulant $\left.K_{2} \equiv\left[d^{2} / d(i s)^{2}\right] \Phi(s)\right|_{s=0}$ and the heat current $[22-24,45] d \hat{Q} / d t=\left(\gamma \hat{\mathcal{V}}-\sqrt{2 \gamma \mathcal{T}} \hat{\xi}_{G}\right) \circ \hat{\mathcal{V}}$ with the product defined in the Stratonovich sense [59]. Remarkably, the direction of heat current is independent of the thermal temperature $\mathcal{T}$, which implies that the athermal environment has high effective temperature under assumptions (i)-(iii). This result is consistent with various experiments [27,33-37], where effective temperatures of athermal noise are reported to be much higher than the room temperature.

We now discuss the physical criteria behind assumption (iii). Let us first expand $W_{\epsilon}(v ; y)$ as $W_{\epsilon}\left(v ; y^{*}\right)=$ $W_{\epsilon}\left(0 ; y^{*}\right)+W_{\epsilon}^{(1)}\left(0 ; y^{*}\right) v+O\left(v^{2}\right) \quad$ with $\quad W_{\epsilon}^{(n)}\left(v ; y^{*}\right) \equiv$ $\partial^{n} W_{\epsilon}\left(v ; y^{*}\right) / \partial v^{n}$ with the typical velocity jump $y^{*}$. The essence of our expansion is that the nonlinear part of $W_{\epsilon}(v ; y)$ is asymptotically irrelevant in the small $\epsilon$ limit as shown in Eq. (6), i.e., $\left|W_{\epsilon}(0 ; y)\right| \gg v\left|W_{\epsilon}^{(1)}(0 ; y)\right|$. We then introduce the nonlinear temperature $T_{\mathrm{NL}} \equiv \mid W_{\epsilon}\left(0 ; y^{*}\right) /$ $\left.W_{\epsilon}^{(1)}\left(0 ; y^{*}\right)\right|^{2} / 2$, which characterizes the relevance of the nonlinear part of $W_{\epsilon}(v ; y)$. Then, assumption (iii) is equivalent to $T / T_{\mathrm{NL}}=O\left(\epsilon^{2}\right) \ll 1$.

Violation of the CLT.-We here discuss the mechanism of the violation of the CLT. According to the CLT, the summation of the independent and identically distributed variables converges to a Gaussian random variable. Since the white noise $\hat{\xi}$ is regarded as independent and identically distributed, the summation $\int_{0}^{t} d s \hat{\xi}(s) / \sqrt{t}$ converges to a Gaussian variable for $t \gg \tau_{P}$, where $\tau_{P}$ is the characteristic time interval between athermal collisions. When the thermal friction is absent, the relaxation time $\tau_{R}$ diverges because the athermal friction is proportional to $\epsilon$ as $\gamma_{A}=-\epsilon \alpha_{1}{ }^{\prime}(0)$, which ensures that the system moves slowly in the time scale of $\tau_{P}$ and the CLT is applicable. In contrast, when the thermal friction is sufficiently strong, $\tau_{R}$ is the same order of $\tau_{P}\left(\tau_{R} \simeq \tau_{P}\right)$. The CLT is not applicable anymore in this situation. The above picture clarifies the mechanisms of the violation of the CLT and the emergence of non-Gaussianity.

Example.-Granular motor under viscous friction. - Let us consider a granular motor under viscous friction [see Fig. 3(a)]. We prepare a rotor of cuboid shape with mass $M$, inertial moment $I$, height $h$, width $w$, and depth $l$. The rotor is immersed in two environments: a viscous fluid and a granular gas. The viscous fluid is a thermal bath characterized by viscous coefficient $\gamma$ and temperature $T$. The granular gas under vertical vibration is a steady athermal bath characterized by velocity distribution function (VDF) $f(\vec{v})$, particle's mass $m$, and restitution coefficient $e$. For simplicity, we assume an exponential granular VDF [60] as $f(\vec{v})=e^{-|\vec{v}| / v_{0}} / 8 \pi v_{0}^{3}$ and $T=l=0$. Note that similar setups under dry friction are experimentally realized in Refs. [31,33-35]. The angular velocity $\hat{\omega}$ is driven by the thermal and athermal forces in the viscous fluid and the granular gas, respectively. We assume that the granular gas is so dilute that the athermal force $\hat{F}_{A}$ can be described by the Boltzmann-Lorentz model [31-35,61-65]. By introducing the mass ratio $\epsilon \equiv m / M$, we obtain the master equation for $\hat{\omega}$ as 


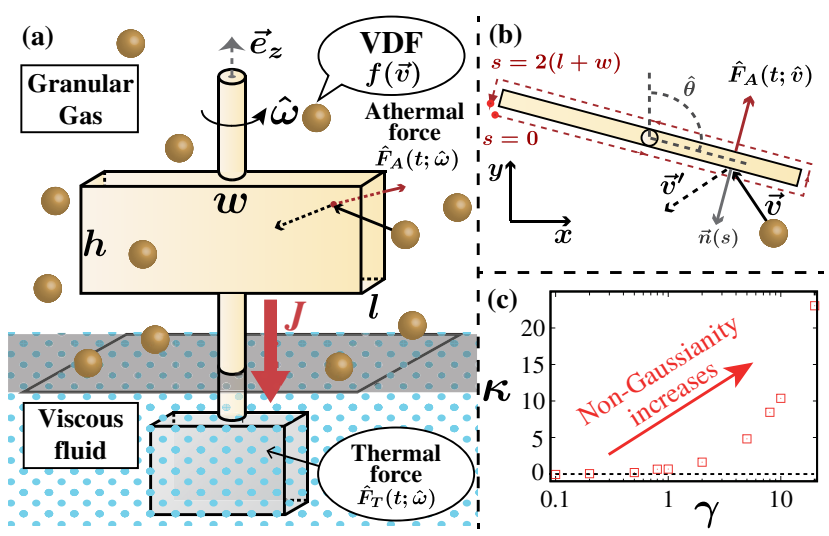

FIG. 3 (color online). (a) Schematic of a rotor driven by the thermal force $\hat{F}_{T}(t ; \hat{\omega})$ from the viscous fluid and the athermal force $\hat{F}_{A}(t ; \hat{\omega})$ caused by collisions of the granular particles. The heat current $J$ flows from the granular gas to the viscous fluid. We fix parameters as $M=I=h=e=\rho=v_{0}=1, T=l=0$, $m=0.01$, and $w=\sqrt{12}$ for numerical simulations. (b) Collisional rules are illustrated. (c) Numerical demonstration of the emergence of $\kappa \equiv\left\langle\hat{\omega}^{4}\right\rangle /\left\langle\hat{\omega}^{2}\right\rangle^{2}-3$ corresponding to the increases of the viscosity $\gamma$.

$$
\begin{aligned}
\frac{\partial}{\partial t} P(\omega, t)= & \gamma\left[\frac{\partial}{\partial \omega} \omega+\frac{T}{I} \frac{\partial^{2}}{\partial \omega^{2}}\right] P(\omega, t) \\
& +\int d y\left[P(\omega-y, t) W_{\epsilon}(\omega-y ; y)\right. \\
& \left.-P(\omega, t) W_{\epsilon}(\omega ; y)\right]
\end{aligned}
$$

where we have introduced the angular velocity PDF $P(\omega, t) \equiv P[\hat{\omega}(t)=\omega]$, the athermal transition rate $W_{\epsilon}(\omega ; y)=\rho h \int d s \int d \vec{v} f(\vec{v}) \Theta(\Delta \vec{V} \cdot \vec{n})|\Delta \vec{V} \cdot \vec{n}| \delta[y-\Delta \omega]$, the coordinate along the cuboid $s$, the normal unit vector to the surface $\vec{n}(s)$, the number density $\rho$, the inertia radius $R_{I} \equiv \sqrt{I / M}, \vec{e}_{z} \equiv(0,0,1), \vec{V}(s) \equiv \omega \vec{e}_{z} \times \vec{r}(s), \Delta \vec{V}(s) \equiv$ $\vec{V}(s)-\vec{v}, \vec{t}(s) \equiv \vec{e}_{z} \times \vec{n}(s), g(s) \equiv \vec{r}(s) \cdot \vec{t}(s) / R_{I}$, and $\Delta \omega \equiv$ $\epsilon g(s)(1+e)[\Delta \vec{V}(s) \cdot \vec{n}] / R_{I}\left[1+\epsilon g^{2}(s)\right] \quad[$ see Fig. 3(b)]. We stress that the granular force is not white noise in general because of the presence of the environmental correlation. Indeed, the athermal transition rate $W_{\epsilon}(\omega ; y)$ depends on $\omega$. We also stress that the non-Gaussianity $\kappa \equiv\left\langle\hat{\omega}^{4}\right\rangle /\left\langle\hat{\omega}^{2}\right\rangle^{2}-3$ is irrelevant for $\epsilon \rightarrow 0$ when the thermal friction is absent $(\gamma=0)$ as shown in Fig. 3(c), although Eq. (9) has no time-reversal symmetry [64,65].

Here, we assume that the mass ratio $\epsilon$ is small and the thermal friction is much larger than the athermal friction (i.e., $\epsilon$ independence of $\gamma$ ). By introducing a scaled variable $\hat{\Omega} \equiv \hat{\omega} / \epsilon$, we obtain the non-Gaussian Langevin equation in the limit $\epsilon \rightarrow+0$ :

$$
\frac{d \hat{\Omega}}{d t}=-\gamma \hat{\Omega}+\hat{\eta}_{g}
$$
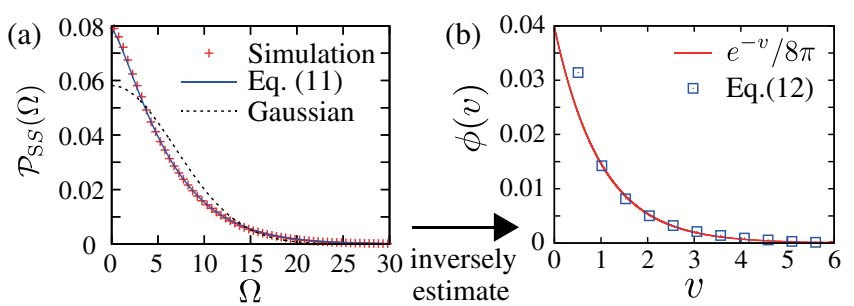

FIG. 4 (color online). (a) Steady PDF of the angular velocity $\hat{\omega}$ obtained from the Monte Carlo simulation of the BoltzmannLorentz equation (9) for $\gamma=2$ (cross points), our theoretical line (11) (solid line), and the conventional Gaussian theory (5) (dashed line). (b) The granular VDF estimated from $\mathcal{P}_{\mathrm{SS}}(\Omega)$ using Eq. (12). Note that the accuracy of the point at $v \simeq 0.5$ is not good because of the singularity in Eq. (12) around $v=0$.

where $\hat{\eta}_{g}$ is the granular collisional torque characterized by the cumulant function $\Phi(s) \equiv-\rho h w v_{0} \Omega_{g}^{2} s^{2}\left(5+3 \Omega_{g}^{2} s^{2}\right) /$ $2\left(1+\Omega_{g}^{2} s^{2}\right)^{2}$ with $\Omega_{g} \equiv w v_{0}(1+e) / 2 R_{I}^{2}$. We then obtain the exact steady PDF for the scaled angular velocity $\tilde{\Omega} \equiv \Omega / \Omega_{g}$ (see the Supplemental Material [55]):

$$
\mathcal{P}_{\mathrm{SS}}(\tilde{\Omega})=\int_{-\infty}^{\infty} \frac{d s}{2 \pi} \frac{e^{\left[-i s \tilde{\Omega}-v_{0} s^{2} / \tilde{v}\left(1+s^{2}\right)\right]}}{\left(1+s^{2}\right)^{3 v_{0} / 2 \tilde{v}}},
$$

where $\tilde{v} \equiv 2 \gamma / \rho h w$. The validity of Eq. (11) is numerically checked by the Monte Carlo simulation of Eq. (9) shown in Fig. 4(a), where the theoretical line perfectly agrees with the numerical data, while the conventional Gaussian model (5) does not. Note that the granular impulses are reduced to the white noise as the environmental correlation disappears (i.e., the athermal force $\hat{\eta}_{g}$ becomes $\hat{\omega}$ independent). Furthermore, the non-Gaussianity becomes relevant as the thermal friction increases as illustrated in Fig. 3(c). We also note that the steady heat current $J=I \gamma\left\langle\hat{\Omega}^{2}\right\rangle>0$ flows from the granular gas to the viscous fluid, which implies that the rotor is far from thermal equilibrium.

We demonstrate the usefulness of the inverse formula (8) to infer the properties of nonequilibrium baths. We assume that the VDF of the granular gas is isotropic: $f(\vec{v})=\phi(|\vec{v}|)$. From Eq. (8), we obtain the following formula for an arbitrary $\phi(v)$ :

$$
\phi(v)=\int_{0}^{\infty} \frac{d s}{\pi|v|}\left[a-\frac{b s^{2}}{2}-c s^{3} \frac{d}{d s} \log \tilde{P}_{\mathrm{SS}}\left(s / F_{g}\right)\right] \cos (s v)
$$

where $a \equiv \int_{-\infty}^{\infty} d v|v| \phi(v), b \equiv \int_{-\infty}^{\infty} d v|v|^{3} \phi(v), c \equiv \gamma / 2 \pi \rho h w$, $F_{g} \equiv w(1+e) / 2 R_{I}^{2}$, and $\tilde{P}_{\mathrm{SS}}(s) \equiv \int_{-\infty}^{\infty} d \Omega e^{i s \Omega} \mathcal{P}_{\mathrm{SS}}(\Omega)$. Equation (12) is applicable to infer the granular VDF from the observation of the rotor's PDF. This implies that the non-Gaussianity in $\mathcal{P}_{\mathrm{SS}}(\Omega)$ is useful to infer the microscopic properties of the athermal bath. Note that the coefficients $a$ and $b$ are determined by the 
Riemann-Lebesgue lemma [57] $\lim _{s \rightarrow \infty}\left[a-b s^{2} / 2-\right.$ $\left.c s^{3}(d / d s) \log \tilde{P}_{\mathrm{SS}}\left(s / F_{g}\right)\right]=0$. We demonstrate the validity of Eq. (12) for $\phi(v)=e^{-|v|} / 8 \pi$ in Fig. 4(b), where $\phi(v)$ is estimated from Eq. (12). This is a clear demonstration of the effectiveness of the inverse formula (12).

The meaning of the inverse formula (12) can be understood from the viewpoint of "cooling" of the rotor. In the absence of a thermal environment, the rotor's effective temperature approaches that of the granular gas. Conversely, in the presence of a thermal environment, the effective temperature is less than that of the granular gas because the thermal environment plays the role of a "cooler." It absorbs redundant information from the rotor's motion, and, therefore, the precise information of athermal noise (i.e., high-order cumulants) is accessible from the rotor's dynamics.

Conclusion.- In this Letter, we have considered a tracer particle attached to both thermal and athermal environments and derived a non-Gaussian Langevin equation (7) subject to the condition that the athermal stochastic force is irrelevant during relaxation. We also derived an inverse formula (8) to infer the environmental information from the observation of the tracer particle. We applied our formulation to a granular motor under viscous friction and analytically obtained the stationary PDF (11) and an inverse formula (12) on the granular velocity distribution.

We revealed the emergence of the non-Gaussianity and its microscopic origins. Extensions of this formulation to multidimensional nonlinear systems are planned for the future. Our theory serves as a foundation of athermal statistical mechanics and would be important for various fields of science, such as biophysics, chemistry, and econophysics.

We are grateful for useful discussion with N. Nakagawa, K. Sekimoto, and A. Puglisi. This work was supported by the Japan Society for Promotion of Science (JSPS) Core-toCore Program "Non-equilibrium dynamics of soft matter and information," Grants-in-Aid for the JSPS Fellows (Grants No. 24.3751 and No. 26·2906), and JSPS KAKENHI Grants No. 25287098 and No. 25800217.

[1] C. Bustamante, J. Liphardt, and F. Ritort, Phys. Today 58, No. 7, 43 (2005).

[2] R. Phillips, J. Kondev, and J. Theriot, Physical Biology of the Cell (Garland, New York, 2008).

[3] J. Liphardt et al., Science 296, 1832 (2002).

[4] D. Collin, F. Ritort, C. Jarzynski, S. B. Smith, I. Tinoco, and C. Bustamante, Nature (London) 437, 231 (2005).

[5] K. Hayashi, H. Ueno, R. Iino, and H. Noji, Phys. Rev. Lett. 104, 218103 (2010).

[6] S. Toyabe, T. Okamoto, T. Watanabe-Nakayama, H. Taketani, S. Kudo, and E. Muneyuki, Phys. Rev. Lett. 104, 198103 (2010).
[7] D. M. Carberry, J. C. Reid, G. M. Wang, E. M. Sevick, D. J. Searles, and D. J. Evans, Phys. Rev. Lett. 92, 140601 (2004).

[8] E. H. Trepagnier, C. Jarzynski, F. Ritort, G. E. Crooks, C. Bustamante, and J. Liphardt, Proc. Natl. Acad. Sci. U.S.A. 101, 15038 (2004).

[9] V. Blickle, T. Speck, L. Helden, U. Seifert, and C. Bechinger, Phys. Rev. Lett. 96, 070603 (2006).

[10] N. Garnier and S. Ciliberto, Phys. Rev. E 71, 060101(R) (2005).

[11] S. Ciliberto, A. Imparato, A. Naert, and M. Tanase, Phys. Rev. Lett. 110, 180601 (2013).

[12] R. van Zon, S. Ciliberto, and E. G. D. Cohen, Phys. Rev. Lett. 92, 130601 (2004).

[13] D. J. Evans, E. G. D. Cohen, and G. P. Morriss, Phys. Rev. Lett. 71, 2401 (1993).

[14] G. Gallavotti and E. G. D. Cohen, Phys. Rev. Lett. 74, 2694 (1995).

[15] G. E. Crooks, Phys. Rev. E 60, 2721 (1999).

[16] C. Jarzynski, Phys. Rev. Lett. 78, 2690 (1997).

[17] U. Seifert, Phys. Rev. Lett. 95, 040602 (2005).

[18] J. Kurchan, J. Phys. A 31, 3719 (1998).

[19] T. Hatano and S.-I. Sasa, Phys. Rev. Lett. 86, 3463 (2001).

[20] T. Harada and S.-I. Sasa, Phys. Rev. Lett. 95, 130602 (2005).

[21] T. Sagawa and M. Ueda, Phys. Rev. Lett. 104, 090602 (2010).

[22] K. Sekimoto, J. Phys. Soc. Jpn. 66, 1234 (1997).

[23] K. Sekimoto, Prog. Theor. Phys. Suppl. 130, 17 (1998).

[24] K. Sekimoto, Stochastic Energetics (Springer-Verlag, Berlin, 2010).

[25] U. Seifert, Rep. Prog. Phys. 75, 126001 (2012).

[26] U. Seifert, Eur. Phys. J. B 64, 423 (2008).

[27] E. Ben-Isaac, Y. Park, G. Popescu, F. L. H. Brown, N. S. Gov, and Y. Shokef, Phys. Rev. Lett. 106, 238103 (2011).

[28] N. Gov, Phys. Rev. Lett. 93, 268104 (2004).

[29] L. C.-L. Lin, N. Gov, and F. L. H. Brown, J. Chem. Phys. 124, 074903 (2006).

[30] É. Fodor, K. Kanazawa, H. Hayakawa, P. Visco, and F. van Wijland, Phys. Rev. E 90, 042724 (2014).

[31] P. Eshuis, K. van der Weele, D. Lohse, and D. van der Meer, Phys. Rev. Lett. 104, 248001 (2010).

[32] J. Talbot, R. D. Wildman, and P. Viot, Phys. Rev. Lett. 107, 138001 (2011).

[33] A. Gnoli, A. Petri, F. Dalton, G. Pontuale, G. Gradenigo, A. Sarracino, and A. Puglisi, Phys. Rev. Lett. 110, 120601 (2013).

[34] A. Gnoli, A Puglisi, and H. Touchette, Europhys. Lett. 102, 14002 (2013).

[35] A. Gnoli, A. Sarracino, A. Puglisi, and A. Petri, Phys. Rev. E 87, 052209 (2013).

[36] J. Gabelli and B. Reulet, Phys. Rev. B 80, 161203(R) (2009).

[37] A. M. Zaklikiewicz, Solid State Electron. 43, 11 (1999).

[38] Y. M. Blanter, and M. Büttiker, Phys. Rep. 336, 1 (2000).

[39] J. P. Pekola, Phys. Rev. Lett. 93, 206601 (2004).

[40] P. Addesso, G. Filatrella, and V. Pierro, Phys. Rev. E 85, 016708 (2012). 
[41] D. Valenti, C. Guarcello, and B. Spagnolo, Phys. Rev. B 89, 214510 (2014).

[42] J. Łuczka, T. Czernik, and P. Hänggi, Phys. Rev. E 56, 3968 (1997).

[43] A. Baule and E. G. D. Cohen, Phys. Rev. E 79, 030103(R) (2009).

[44] W. A. M. Morgado and S. M. Duarte Queiros, Phys. Rev. E 86, 041108 (2012).

[45] K. Kanazawa, T. Sagawa, and H. Hayakawa, Phys. Rev. Lett. 108, 210601 (2012).

[46] K. Kanazawa, T. Sagawa, and H. Hayakawa, Phys. Rev. E 87, 052124 (2013).

[47] K. Kanazawa, T. Sagawa, and H. Hayakawa, Phys. Rev. E 90, 012115 (2014).

[48] J. Klafter and I. M. Sokolov, First Steps in Random Walks: From Tools to Applications (Oxford University Press, New York, 2011).

[49] R. Metzler and J. Klafter, Phys. Rep. 339, 1 (2000).

[50] R. Klages, G. Radons, and I. M. Sokolov, Anomalous Transport: Foundation and Applications (Wiley-VCH, Weinheim, 2008).

[51] N. G. van Kampen, Can. J. Phys. 39, 551 (1961).
[52] N. G. van Kampen, Stochastic Processes in Physics and Chemistry, 3rd ed. (North-Holland, Amsterdam, 2007).

[53] R. Zwanzig, J. Stat. Phys. 9, 215 (1973).

[54] C. De Bacco, F. Baldovin, E. Orlandini, and K. Sekimoto, Phys. Rev. Lett. 112, 180605 (2014).

[55] See Supplemental Material at http://link.aps.org/ supplemental/10.1103/PhysRevLett.114.090601 for details.

[56] I. Eliazar and J. Klafter, J. Stat. Phys. 111, 739 (2003).

[57] R. Strichartz, A Guide to Distribution Theory and Fourier Transforms (World Scientific, Singapore, 2008).

[58] S. Albeverio and B. Smii, arXiv:1312.2324v1.

[59] C. Gardiner, Stochastic Methods, 4th ed. (Springer-Verlag, Berlin, 2009).

[60] J. S. Olafsen and J. S. Urbach, Phys. Rev. E 60, R2468 (1999).

[61] H. A. Lorentz, Proc. Amst. Acad. 7, 438 (1905).

[62] G. Gallavotti, Phys. Rev. 185, 308 (1969).

[63] H. Spohn, Commun. Math. Phys. 60, 277 (1978).

[64] J. J. Brey, M. J. Ruiz-Montero, R. García-Rojo, and J. W. Dufty, Phys. Rev. E 60, 7174 (1999).

[65] N. V. Brilliantov and T. Pöschel, Kinetic of Theory of Granular Gases (Oxford University Press, New York, 2003). 